\title{
Lessons from Implementing a Mass Vaccination Campaign in a Highly-Urbanized City in the Philippines
}

\author{
Carl Abelardo T. Antonio ${ }^{1,2}$ and Teresita G. Hilario ${ }^{1}$ \\ ${ }^{1}$ Pasay City Health Office, Pasay City \\ ${ }^{2}$ College of Public Health, University of the Philippines Manila
}

\begin{abstract}
Objective. To document the processes and outcomes of a measles-rubella supplemental immunization activity implemented in Pasay City, Philippines.

Methods. Review of proceedings of meetings, memoranda, manuals, and reports. Interviews with field monitors, supervisors and vaccination teams, and key members of the project technical group.

Results. A total of 60,685 children age 9 to $>72$ months were reached during the campaign, representing $75 \%$ of the estimated eligible population. On the other hand, 1,453 children were unimmunized because of [a] scheduled or documented prior immunization with a measles-containing vaccine $(n=$ $1,154)$, and [b] refusal of parents due to religious or cultural reasons $(n=237)$. The successful completion of the measlesrubella supplemental immunization activity (MR-SIA) was grounded on appropriate timing of project implementation; a clearly identified problem; the existence of linkages between stakeholders at the national and sub-national level; strong local chief executive support; the availability of resources to support project implementation; a clear policy statement; and implementation guided by an organizational structure and tailored project plans.
\end{abstract}

Conclusion. In this paper, we have shown that the success of public health programs and projects rely not only on technical soundness of the intervention or adequate resource mobilization, but require wide grassroots support that is guided by an able leadership and clear policy.

Key Words: mass vaccination, measles-rubella vaccine, Philippines

\section{Introduction}

Measles is a highly contagious disease of viral etiology, affecting primarily children under five years of age. Despite the availability of a safe and effective vaccine, deaths attributable to measles remain among the leading causes of mortality worldwide.

\footnotetext{
Corresponding author: Carl Abelardo T. Antonio, MD, MPH (cand) Office of the City Health Officer

City Government of Pasay

Room 117 City Hall, F.B. Harrison St., Pasay City 1302 Philippines

Telefax: +6328318201

Email: ctantoniomd@gmail.com
}

In 2008, for instance, 164,000 measles deaths were reported globally, with $95 \%$ of cases occurring "in lowincome countries with weak health infrastructures". ${ }^{1}$ In the same year, the Philippines reported 1,331 measles cases and seven deaths [Case Fatality Rate, CFR, 0.53\%]. About one in four cases $(26 \%)$ reported were from the National Capital Region (NCR). ${ }^{2}$

While there was an observable decline in measles morbidity and mortality both locally and globally, case reports of laboratory-confirmed measles indicate an uninterrupted transmission of the virus, placing at risk unprotected individuals. This becomes of greater concern when seen through the context of inadequate immunization coverage: routine measles immunization in the country remains below the $95 \%$ target coverage. ${ }^{3}$

Congested living spaces, coupled with high population mobility, accumulation of susceptible children because of low immunization coverage for measles particularly in the past year, and a resurgence of laboratory-confirmed measles cases among the unimmunized, provide an environment conducive for uninterrupted transmission of the measles virus and, consequently, a condition ripe for an outbreak to occur.

The Philippines, on the other hand, has committed itself to the fourth Millennium Development Goal (MDG 4) of reducing under-five mortality rate by two-thirds between 1990 and 2015.,5 The goal, therefore, of eliminating measles as a public health problem, a key MDG indicator, requires concerted and intensified action at multiple fronts.

One strategy to attain this end is the conduct of supplemental immunization activities (SIAs), which provide a 'second opportunity' for measles immunization to [a] eliminate any measles susceptibility that has developed in recent birth cohorts, and [b] protect children who did not respond to their first measles vaccination. ${ }^{6}$

The significant coverage (i.e. more than 95\%) reported during the previous mass measles immunization campaigns initiated by the Philippine Department of Health (DOH), as well as the absence of measles cases reported within the succeeding period, lends support to this strategy. ${ }^{7}$

In this paper, we report on process and outcomes of the measles-rubella supplemental immunization activity (MRSIA) conducted in Pasay City from November 2010 to May 
2011. We also discuss project strengths and limitations to guide implementation of similar activities in the future.

\section{Methods}

Context. Pasay City is a fast-rising highly urbanized city in Metro Manila, Philippines with a population of 413,885 as of 2010. The City has a total land area of 18.50 square kilometres, of which only $34.25 \%$ is devoted to residential use, making Pasay City one of the most densely populated areas in NCR. Known as the Premiere Gateway to the Philippines, the City hosts major terminals of all transport types - national and domestic airports, sea ferry boats, and bus and light train terminals.

Prevention and control of measles forms part of the functions Pasay City Health Office (PCHO) through two core programs: [a] provision of vaccines in all 14 health facilities under the Expanded Program on Immunization (EPI); ${ }^{9}$ and [b] disease surveillance by the City Epidemiological and Surveillance Unit (CESU) through reports provided by 14 local primary care health facilities and selected sentinel hospitals (as part of the Philippine Integrated Disease Surveillance and Response, PIDSR). ${ }^{10}$

During the past decade, the number of reported and confirmed measles cases in Pasay City has considerably declined, owing primarily to consistently high immunization coverage (i.e., more than $95 \%$ ).

Meanwhile, Pasay City has consistently distinguished itself as a capable local government unit (LGU) committed to furthering the health of peoples through innovative programs and projects that harness the collaborative capacities of different government agencies, communitybased organizations, development partners and the academe.

It is within this context that the MR-SIA was implemented.

Implementation Strategy. The current supplemental immunization activity, dubbed Ligtas sa Tigdas ang Pasay, is a 20-day door-to-door immunization drive for measles and rubella among children age 9 to 95 months at the time of the campaign who have not received any measles-containing vaccine within the previous four weeks.

To reach as many eligible children as possible, a strict door-to-door immunization strategy was implemented for this SIA, with doors referring not only to those of houses, condominiums, apartments, tenements, orphanages and halfway homes, but also of non-conventional doors in the community, to wit:

- Informal settlements, i.e. families/persons living under bridges; inside parks, cemeteries and open spaces; in tents, carts, abandoned buildings, old vehicles/trans/motorboats; under trees; in islands in the middle of streets, etc.

- All business establishments and market stalls
In contrast with the previous SIAs, Ligtas sa Tigdas ang Pasay entails certification of a "Measles-Free Pasay City", which is part of a nationwide strategy to declare the Philippines measles-free in line with international goals.

A measles-free certification will be granted if the following criteria are met during the certification process at the end of the campaign, to wit:

1. All barangays have passed the Rapid Coverage Assessment (RCA) with no missed child and $>95 \%$ house marking accuracy; AND

2. There are no measles cases for the next three months after the campaign; AND

3. Measles surveillance indicators have met the national standard*.

Methods. Proceedings of meetings, memoranda, manuals, and reports were reviewed to document the processes in the planning and implementation of the MRSIA, using the World Health Organization (WHO) health systems framework as guide. 8,11 Supplemental information was also sourced from interviews with field monitors, supervisors and vaccination teams, as well as key members of the MR-SIA technical group.

The MR-SIA daily and weekly health facility reports filed with the task force, as well as consolidation reports from $\mathrm{PCHO}$, were reviewed to determine coverage statistics. These documents were validated by the duly designated supervisors and monitors, and the MR-SIA technical team.

\section{Results and Discussion}

\section{Leadership: Local Government Support}

A key initial step in the development and implementation of the MR-SIA was securing the City Government's support, through the Hon. Antonino G. Calixto, City Mayor.

Executive Order (EO) No. 14-2010, An Executive Order Establishing a Supplemental Immunization Activity for a Measles-free Pasay City Better Known as the 'Ligtas sa Tigdas ang Pasay Program' and for Other Purposes, signed on 18 October 2010, [a] formalized the incumbent leadership's commitment to the goal of eliminating measles as a public health threat, and [b] mobilized local government agencies, barangay officials, and other sectors to actively participate in the campaign and, thus, ensure that all eligible children are vaccinated during MR-SIA.

\footnotetext{
* These national standards include: [a] At least $80 \%$ of surveillance should report each week on the presence or absence of suspected measles cases; [b] At least $80 \%$ of the reported suspected cases should be reported within 48 hours of rash onset; [c] At least $80 \%$ of the reported suspected cases should be investigated within 48 hours of report; [d] At least $80 \%$ of specimens should be taken from initial contact until 28 days post-rash onset and reach the laboratory in a suitable state for testing; and [d] At least $80 \%$ of specimens must be tested and the result reported back to the surveillance unit within seven days of receipt of the specimen in the laboratory.
} 
Among the agencies mentioned in the EO are the Department of Education (DepEd) Division of City Schools in Pasay City, PCHO, City Social Welfare and Development Office (CSWD), and the Barangay Action Center (BAC).

Service Delivery: Planning, Supervision, Monitoring and Evaluation

Planning for the MR-SIA occurred at several levels. While the $\mathrm{DOH}$ and WHO developed the protocol and manual of procedures for the supplemental immunization drive, each health center area was tasked to develop a microoperational plan that would enunciate concrete, step-by-step measures that will be undertaken by the health staff for information dissemination, social mobilization, community stakeholder participation, and local level monitoring and supervision within the 20-day SIA. Identification of target population, determination of supplies requirement, and scheduling of vaccination among the different barangays was also documented in the operational plan.

All these data were integrated into the city-wide operational plan, in addition to identification of monitoring and evaluation schedule and strategies, data collection measures, management of adverse event following immunization (AEFI), and healthcare waste disposal.

Supervision of vaccination teams for each health center area was delegated to their respective physicians-in-charge, while monitoring was done by a duly designated PCHO supervisory staff or DOH technical staff.

The supervisor was tasked with ensuring that [a] all vaccination teams adhered to the manual of procedures for the MR-SIA as well as the health center operational plan, [b] adequate supplies were available at the health center level at any given time, [c] community stakeholders were mobilized, [d] Vaccination teams (VTs) practiced safe immunization in the field, [e] missed children were revisited, and [f] possible cases of AEFI issues and concerns were raised and discussed with VTs, the designated monitor, and the SIA technical group.

The monitor, on the other hand, was tasked with [a] validating immunization coverage within the health center area, $[\mathrm{b}]$ coordinating with the technical group the schedule of RCA, and [c] serving as liaison between health center and technical team.

Supervision was carried out on a daily basis throughout the 20-day campaign, with meetings between supervisors and VTs held twice daily: once prior to, and another immediately after, VTs' rounds in the community (documented in Reporting Form 2, Highlights of Daily Meetings). Monitoring, on the other hand, was done at least twice weekly (depending upon the availability of the designated monitor).

Weekly meetings of supervisors, monitors and technical team members were also held to discuss issues encountered by VTs, identify potential threats to complete coverage of all eligible children, share good practices that facilitated saturation of some areas, and develop appropriate interventions for project implementation.

Process evaluation was carried out [a] daily at the health center level between VTs and supervisor and/or monitor (if available), [b] weekly at the city level between supervisors, monitors and the SIA technical team, and [c] at the end of the 20-day campaign.

Evaluation of vaccination coverage was done by a duly designated team of WHO and DOH staff once a health center supervisor and monitor indicated that a barangay is ready for rapid coverage assessment using the guidelines mentioned above.

\section{Health Workforce}

The MR-SIA employed a novel methodology pertaining to [i] identification of eligible children, [ii] scope of door and household within the campaign, [iii] data recording and reporting, [iv] house marking, and [v] monitoring and evaluation (these are discussed elsewhere in this report). Thus, training of field health workers prior to the commencement of the project became a priority.

A series of orientation-seminars for all PCHO personnel were held by WHO and DOH technical staff on October 1213, 14-15, and 26-27, 2011 to introduce monitors, supervisors and vaccinators to the MR-SIA strategy. A manual of procedures was also published to supplement the training provided.

Based on estimates, it was determined by the technical committee that a total of 54 VTs were required to completely saturate all barangays of Pasay City within the 20-day campaign period, with each VT composed of a vaccinator, recorder and guide ${ }^{t}$. At the outset, a total of $162 \mathrm{PCHO}$ personnel and barangay health workers were mobilized for the MR-SIA. Towards the end of the campaign, the number of MR-SIA field staff almost doubled.

Eighty-eight vaccination teams comprised of $320 \mathrm{PCHO}$ staff, barangay health workers and volunteer paramedical personnel were mobilized for MR-SIA to saturate all 201 barangays.

In addition, $14 \mathrm{DOH}$ and $\mathrm{PCHO}$ technical staff were appointed monitors, while 18 physicians were made health center supervisors. A total of 516 and 270 visits during the 20-day campaign were made by monitors and supervisors, respectively.

\section{Information: Data Management}

Each VT was provided with three recording forms: a daily accomplishment log (Recording Form 1, Vaccination Team's Daily Accomplishment) detailing the number of

\footnotetext{
+ This estimate was based on the following assumptions: [a] There are 81,038 children age 9 to 95 months (or $19.27 \%$ of the total population) who will have to be vaccinated; [b] Each vaccination team can cover a minimum of 75 children per day; and [c] All teams will be working for 20 days.
} 
households visited, number of children given measles-rubella (MR) vaccine per age cohort ${ }^{\ddagger}$, and number of vaccine vials consumed; a line list of missed children (Recording Form 2, List of Households for Revisits); and a line list of children whose parents refused immunization because of documented receipt of measles vaccine from a private medical provider (Recording Form 3, Line List of Eligible Children with Private MDs).

Health center-level data pertaining to the number of children vaccinated (total and per age cohort) and households visited were submitted daily before the close of office hours to the CESU through messenger, phone call or short-messaging system (SMS).

A more detailed weekly report per barangay (Reporting Form 1, Consolidated Accomplishment Report) was also submitted to the CESU every Monday containing all data in Recording Form 1 plus the following indicators: total time to vaccinate, total number of supervisory and monitoring visits, total number of missed children, total number of missed children revisited and given vaccine, number of households with inaccurate markings, and unsafe immunization practices identified, if any.

All data were validated at the health center and $\mathrm{PCHO}$ levels, and were consolidated weekly for presentation during the weekly monitoring meetings.

\section{Medical Products: Logistics and Supplies}

Procurement of supplies (i.e. vaccines, syringes, epinephrine, etc.) and collaterals (i.e. recording and reporting forms, house stickers, immunization cards, other stationery, information materials, etc.) were done by the WHO and DOH. Weekly bulk deliveries were made at the PCHO Central Pharmacy. Distribution to the various health center areas was accomplished at least twice weekly.

Integrity of cold chain from transport, delivery, distribution and dispensing of vaccines was ensured by respective personnel in charge.

Inventory of supplies were done daily at facility level, and weekly at city level.

Safety collection boxes for sharps and used vials and ampoules were provided each VT. These were pooled in locked cabinets or rooms at the facility level for collection at the end of the week by a duly designated team of sanitarians. Collected healthcare waste were brought to Pasay City General Hospital (PCGH) for final disposal.

\section{Financing}

Funding for this project was provided by the WHO (principally for supplies, training expenses, and per diems of vaccination teams), with augmentation from $\mathrm{DOH}$ (for per diems of additional volunteers).

\footnotetext{
₹ For purposes of data collection and analysis, three age cohorts were constructed: [a] 9-11 months, [b] 12-23 months, and [c] 24-95 months.
}

\section{Immunization Coverage}

A total of 60,685 children age 9 to $>72$ months living in 96,443 households in the city's 201 barangays were reached during the current supplemental immunization activity, representi ng $75 \%$ of the estimated eligible population. Older children between 2 and 5 years of age comprised $79 \%$ of eligible population seen (Figure 1).

In so far as the estimated eligible population is concerned, no health center was able to achieve the $100 \%$ target for the MR-SIA, only one (San Pablo) reported an immunization rate higher than the routine immunization target of $95 \%$, and only seven had rates greater than the $77 \%$ reported national measles vaccine coverage in $2009 .{ }^{12}$

As of end of May 2011, 1,453 children were identified as "missed" by the vaccination teams, nearly $80 \%(n=1,154)$ of whom were not vaccinated because of scheduled or documented prior immunization with a measles-containing vaccine with a private healthcare provider; about 1 in 6 $(16 \%)$ parents refused to have their child immunized on religious or cultural grounds (Figure 2). $\S$

\section{Adverse Events Following Immunization}

All VTs were trained on recognition and management of AEFIs, using as basis DOH Administrative Order No. 2010$0017 .{ }^{13}$ To enable rapid response in the field, each vaccinator was provided with two ampoules of epinephrine for use in cases of anaphylaxis. Parents were also instructed on adverse events and were instructed to immediately notify the health center of any occurrence and/or to seek emergency treatment at a referral hospital.

Standing agreements with the four hospitals located in Pasay City - PCGH, San Juan de Dios Educational Foundation, Inc. (Hospital), Manila Adventist Medical Center (MAMC), and Air Force General Hospital (AFGH) as well as the Doña Marta Lying-in Clinic (DMLI) were established for referral and management of probable cases of AEFI.

While reporting AEFIs has already been made mandatory by the DOH, AEFI surveillance was integrated as a distinct MR-SIA activity. ${ }^{13}$ In compliance with existing health policies, health center supervisors were required to report immediately any incidence of AEFI within their area using Reporting Form 3 (Line List for Adverse Events Following Immunization).

No adverse events following immunization were reported in any barangay during, and immediately after, the campaign. 


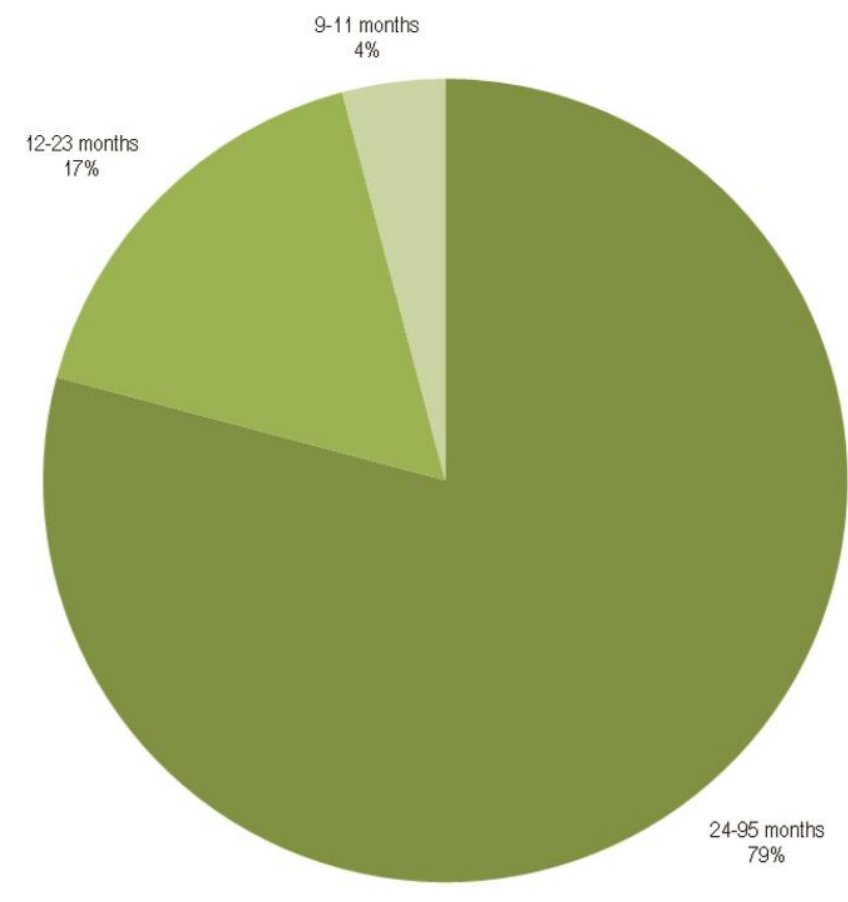

Figure 1. Age distribution of children vaccinated during the MR-SIA, November 2010 to May 2011, Pasay City, Philippines $(n=60,685)$

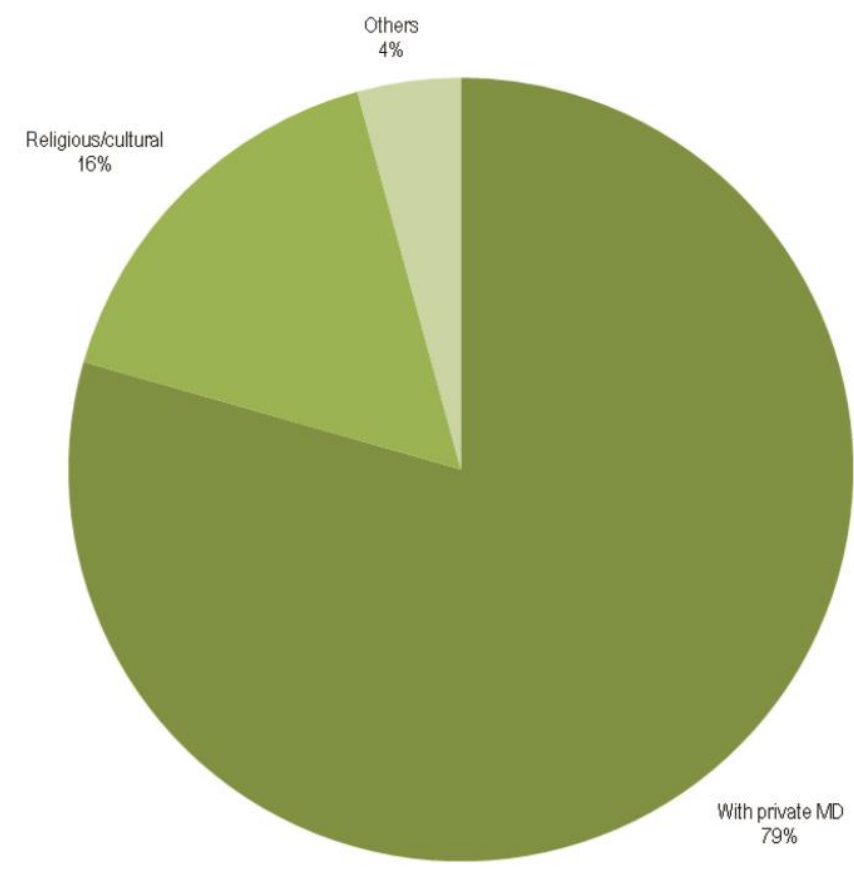

Figure 2. Reasons why some eligible children were not given MR vaccine during the MR-SIA, November 2010 to May 2011, Pasay City, Philippines $(n=1,453)$.

\section{Measles Surveillance}

Reports of measles cases and deaths from all 14 health centers and four hospitals in Pasay City, as well as other sentinel sites under the PIDSR, were monitored by the CESU. Within the three-month period from January to March 2011, seven suspected measles cases were reported: two were determined to be suffering from another medical condition, three were outside the MR-SIA age cohort, while two children were not vaccinated during the SIA (in the first case, parents refused to have the child vaccinated during the MR-SIA because of documented receipt of measlescontaining vaccine from a private physician; second case was a migrant from Leyte who arrived in Pasay City in January 2011 and was, thus, not vaccinated during the MRSIA. This child was subsequently confirmed to be suffering from measles).**

\section{Preliminary RCA Results}

Results of the rapid coverage assessment showed that, except for 20 remaining barangays being deliberated upon by the $\mathrm{WHO}$ and $\mathrm{DOH}$ validating team, all areas in Pasay City have met the minimum criteria for certification and have thus been declared "Passed" by the WHO and DOH technical teams.

As of May 2011, Pasay City has technically completed all requirements for certification laid out in the manual of procedures for the MR-SIA. Pending completion of deliberation of the certification status of three remaining areas, Pasay City is on its way of finally and formally being certified as a measles-free city, a distinction that will be the first in the Philippines and the entire Western Pacific Region.

\section{Lessons Learned: Policy and Practice Implications}

Supplemental immunization activities are support interventions to the routine immunization program (i.e., daily scheduled vaccination in health facilities) and are designed to provide catch-up vaccination for susceptible individuals in the community. ${ }^{6}$ Wide immunization coverage as determined by RCA, and absence of measles cases detected through routine disease surveillance indicate that the MR-SIA was a success.

Based on the project process presented above, we now ask, what are the strengths and weaknesses of project planning and implementation and what are its implications for policy and practice? We use the policy circle framework described by Hardee et $\mathrm{al}^{14}$ in addressing this question.

The project was launched in the context of heightened public awareness regarding measles, social pressure on governments to address the burden of disease, and the existence of, and local experience with, known cost-effective interventions to address the problem. In the months leading

\footnotetext{
** This incident, according to Mercado and Hilario, "represented the extremely problematic task of ensuring full immunization coverage and protection in a city with a population as highly mobile as Pasay City. Nevertheless, vaccination drives such as SIA provide opportunities to assess and accordingly improve existing surveillance systems, laboratory test facilities and capabilities and community awareness of these vaccine-preventable illnesses and other notifiable diseases." (11)
} 
to the campaign, media reports ${ }^{15,16,17}$ were filled with news of outbreaks of measles cases in the country. As was previously mentioned, the Philippines has committed itself to the eradication of measles as a public health threat by 2015, and has previously implemented successful supplemental immunization activities.

Strong linkages between the stakeholders at the national (i.e., between $\mathrm{WHO}$ and $\mathrm{DOH}$ ) and sub-national levels (i.e., between $\mathrm{WHO}, \mathrm{DOH}$ and the Pasay City government; also, among the various agencies and organizations within the city) were already established from previous cooperation, and this facilitated dialogue and project planning and implementation. In the campaign, $\mathrm{WHO}$ and $\mathrm{DOH}$ teams were consistently providing technical assistance to the PCHO technical team in the implementation of the MR-SIA despite commitments in their individual offices. Barangay officials pledged, and provided, to VTs guards and additional guides during daily rounds in their areas. Barangay health workers, student affiliates, and civil society organizations were mobilized to provide assistance to vaccination teams.

It is also worth noting that the local chief executive of Pasay City was very enthusiastic and wholehearted in his support for the campaign. Request of assistance from barangay officials and other agencies, and even modification of health center service schedule to give way to the MR-SIA were facilitated through the local chief executive's support and commitment to the project.

Resources from within, and external to Pasay, were readily available and mobilized for the project. The human resource complement from $\mathrm{WHO}, \mathrm{DOH}, \mathrm{PCHO}$, and other agencies were deployed in the various areas during the duration of the campaign to provide technical and operational support. In addition to project funding from WHO, the LGU has also appropriated funds for provision of transportation and subsistence allowance for MR-SIA participants.

With respect to the policy instrument, the executive order issued by Mayor Calixto set the tone for the LGU's commitment and resolve to see the project through its completion.

The technical/implementation policy, on the other hand, was embodied in the manual of procedures for the MR-SIA made available by the WHO and $\mathrm{DOH}$, as well as the citywide operational plan developed by the PCHO technical team. In addition, detailed, health center-level microoperational plans tailored the implementation of the MR-SIA within their respective contexts, taking into consideration such things as an area's population density, terrain, distance from the health facility, level of support from local officials, and prior experience with people's reception of similar health campaigns.

Involved in the MR-SIA are operations and technical staff from the $\mathrm{PCHO}, \mathrm{DOH}$, and $\mathrm{WHO}$, as well as barangay health workers and hired project staff. The (eclectic) mix of personalities from different agencies with different cultures would definitely result in a clash of ideologies and work practices. The clear line of authority (Figure 3) established from the vaccination team to the city level ensured that all personnel involved worked towards attaining the goal of making each area measles-free within the confines of the MR-SIA procedure and acceptable clinical practice. This chain of command also facilitated resolution of issues and concerns confronted by vaccination teams, supervisors and monitors in the implementation of MR-SIA, but which may not have been adequately covered by existing guidelines.

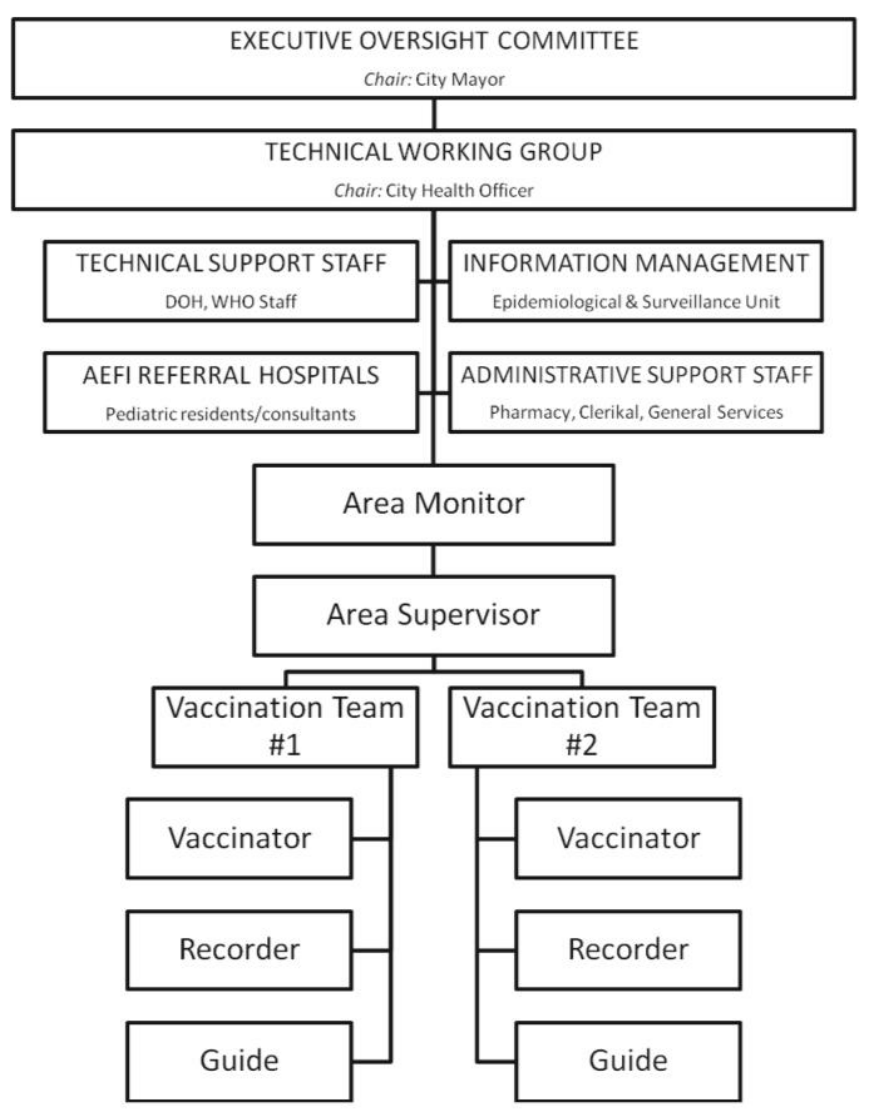

Figure 3. Schematic diagram of the organization structure adopted by the implementors during the MR-SIA, November 2010 to May 2011, Pasay City, Philippines

Monitoring and evaluation of performance at the health center level and city-wide were conducted regularly (daily at the health center, weekly city-wide) to assess progress and identify issues that may potentially thwart any attempt at securing $100 \%$ immunization coverage in any and all areas. As a result, vaccination teams, supervisors and monitors became adept not only in coverage assessment; they also developed problem solving skills that required innovation, ingenuity and an arsenal of adequate and accurate information, to deal with refusal or even resistance to 
immunization - an environment that is very much different from the willing (sometimes captive) audience they usually serve in health facilities.

In summary, the successful completion of the MR-SIA was grounded on appropriate timing of project implementation; a clearly identified problem; the existence of linkages between stakeholders at the national and subnational level; strong local chief executive support; the availability of resources to support project implementation; a clear policy statement; and implementation guided by an organizational structure and tailored project plans.

Replication of similar activities in the future should be guided by a careful consideration of some areas of concern encountered in the present campaign.

The MR-SIA manual of procedures was developed using as basis the wealth of experience gained from previous SIAs (including similar certification activities). Foresight, however, was not sufficient to predict the diverse, and often shifting, sociocultural environments in which the MR-SIA was finally implemented. Hence, as VTs, supervisors and monitors did their daily rounds in the field, novel barriers and roadblocks emerged, while ingenious solutions were developed. These were subsequently shared during weekly meetings, and some ideas were incorporated and adopted into the MR-SIA protocol. While we recognize the birth pains associated with innovations, the parallel evolution of the manual of procedures with progress of MR-SIA meant differing degrees of implementation. For instance, some VTs diligently revisited some missed children in order to comply with the $100 \%$ saturation requirement for certification. After the second week of implementation, the technical team announced that RCA will consider situations in which a child was not vaccinated even after, at most, two revisits.

While the MR-SIA implemented definite criteria for certification, which is based on results of RCA and measles surveillance, estimation of target population and determination of extent of coverage is nonetheless based on population projections provided by the National Statistics Office (NSO). The dissonance between the computed measles-rubella immunization coverage (i.e. all below target) and the result of the RCA (i.e. with the exclusion of 13 barangays, all areas passed) should prompt policymakers to review existing guidelines regarding the use of population projections (as opposed to actual counts) in program evaluation. The availability of local population data, as well as the regularity of the conduct of censuses by the NSO, should be factored into consideration.

In all areas, vaccination teams were most often immediately entertained by individuals and families who have accessed, or are regularly accessing, health center services. The opposite is true with regards those who have private healthcare providers, as borne out by the 1,154 children whose parents refused the offered immunization. While, on the one hand, this is an exercise of a patient's right to choose a healthcare professional, the more troubling issues that need to be confronted are the people's (and sometimes, private physicians') misperceptions regarding the vaccines available in public facilities (i.e. substandard) and the personnel providing the injection (i.e. unskilled).

Certain religious sects expressly forbid injection of any medication to the body. Children may not always be under the care of a knowledgeable and competent caregiver during regular work hours, necessitating revisits at night or even on weekends. Unconventional families and settings exist: children transferred between grandparents' residences; an entire family (wife and children) brought along by a driverfather in his jeepney during work; a two-year old child locked alone inside a house while the parents are working; dark corridors, steep staircases, narrow alleyways - all of these may impede a vaccination team's progress.

Suspicious looks greeted most vaccination teams, particularly among the more affluent neighbourhoods. The reason: mistaken identity - [a] a certain politician's staff (because of uniform color), [b] returning tax collectors (they also wore shirts with the City's seal embossed in the breast pocket). In other situations, people failed to identify the health staff simply because they were not aware of the project. While social mobilization activities were held and information materials were made available, the interval from the time of project promotion to actual implementation proved too short to allow people to became sufficiently aware of, and identify with, MR-SIA; thus, the difficulties encountered. It must be emphasized, however, that other government agencies (i.e. CSWD, DepEd, BAC) and nongovernmental and faith-based organizations generously offered their assistance to the PCHO in reaching as wide an audience as possible for the campaign. For instance, announcements about the MR-SIA were made in Roman Catholic churches several Sundays prior to the campaign. The Church of Jesus Christ of Latter Day Saints as well as the Philippine National Red Cross mobilized their personnel to inform their constituents about the MR-SIA.

Despite the birth pains, however, it goes without saying that the MR-SIA benefited the system (first implementation of a certification-based SIA in the Philippines), healthcare workers (training, experience, community rapport, interaction with other healthcare workers), the community (intersectoral participation), and the population at large (widespread immunization, heightened awareness, decreased incidence of measles). Meanwhile, the project has been replicated nationwide through the Iligtas sa Tigdas ang Pinas campaign, using as springboard the lessons gleaned from the arduous Pasay City experience.

Supplemental immunization activities, however, are indubitably resource-intensive when compared to routine immunization and form just one component of the bigger measles elimination strategy. Measles vaccine is widely available in different government health facilities 
nationwide. ${ }^{9}$ Routine provision of a second dose of measles vaccine (as measles-mumps-rubella, MMR, vaccine) for children age 12 to 15 months was recently integrated into the EPI. ${ }^{7}$ Health workers now face the challenge of [a] strengthening routine immunization, $[b]$ intensifying disease surveillance measures, and [c] raising people's awareness of measles as a preventable disease. The entire health system must also realize that the high mobility afforded by developments in transportation now mean that measles, along with other communicable diseases, is a collective problem of peoples everywhere. Only then can we truly achieve a measles-free Philippines.

\section{Conclusion}

In this paper, we have shown that the success of public health programs and projects rely not only on technical soundness of the intervention or adequate resource mobilization, but require wide grassroots support that is guided by an able leadership and clear policy.

\section{Acknowledgements}

This paper is an original publication of the Pasay City Health Office. The authors acknowledge the support provided by Hon. Antonino G. Calixto, City Mayor, and Dr. Cesar F. Encinares, City Health Officer, in the production of this work.

Potential conflict of interest: CTA is a medical officer, Pasay City Health Office; TGH is a nurse supervisor, Pasay City Health Office.

\section{References}

1. WHO. WHO Fact Sheets: Measles. World Health Organization. [Online]. December 2009. [cited 2011 July]. Available from http://www.who.int/mediacentre/factsheets/fs286/en/.

2. National Epidemiology Center. The Philippine Integrated Disease Surveillance and Response (PIDSR) Annual report 2008. Manila: National Epidemiology Center, Department of Health, 2008.

3. National Epidemiology Center. Field Health Services Information System Annual 2007. Manila: National Epidemiology Center, Department of Health, 2007.

4. DOH. National Objectives for Health 2005-2015. Manila : Department of Health, 2005.

5. United Nations. The Millennium Development Goals Report. New York : United Nations, 2008.

6. Measles Initiative. Global elimination of measles: Report by the Secretariat. Measles Initiative.Org. [Online]. [cited 2011 July]. Available from http://www.measlesinitiative.org/mi-files/Reports/ Measles\%20Mortality\%20Reduction/Eradication/Microsoft $\% 20$ Word $\% 2$ 0-\%20Global\%20measles\%20elimination_EB_Final_060309.pdf.

7. DOH. DOH Department Memorandum 2010-0161, Administration of routine second dose measles-containing vaccine for children. Manila, Philippines: Department of Health, July 01, 2010.

8. WHO. Everybody's business: Strengthening health systems to improve health outcomes. WHO's framework for action. Geneva: World Health Organization, 2007. ISBN 9789241596077.
9. DOH. DOH Administrative Order No. 39, s. 2003, Policies on Nationwide Implementation of the Expanded Program on Immunization. Manila, Philippines: Department of Health, April 21, 2003.

10. DOH. DOH Administrative Order No. 2007-0036, Guidelines on the Philippine Integrated Disease Surveillance and Response (PIDSR) framework. Manila, Philippines: Department of Health, October 1, 2007.

11. Mercado VJ, Hilario T. Ligtas sa Tigdas and Pasay: A process documentation of the supplemental immunization activity for a measles-free Pasay City. 2011. Exit report for the urban rotation under the Straight Internship in Family and Community Medicine program, University of the Philippines College of Medicine and Philippine General Hospital.

12. National Epidemiology Center. Field Health Services Information System Annual Report 2009. Manila: National Epidemiology Center, Department of Health, 2009. Preliminary Report.

13. DOH. DOH Administrative Order No. 2010-0017, Guidelines in Surveillance and Response to Adverse Events Following Immunization (AEFI). Manila, Philippines: Department of Health, June 18, 2010.

14. Hardee K, Feranil I, Boezwinkl J, Clark B. The Policy Circle: A Frameworkfor Analyzing the Components of Family Planning, Reproductive Health, Maternal Health, and HIV/AIDS Policies. POLICY Working Paper Series No. 11. Policyproject.Com. [Online]. [cited 2012 July]. Available from http://www.policyproject.com/pubs/ workingpapers/wps-11.pdf

15. Crisostomo S. DOH warns of measles outbreak. Philstar.Com. [Online]. February 23, 2010 [cited 2012 August]. Available from http://www.philstar.com/Article.aspx?articleid $=552150$

16. Esplanada JE. $189.1 \%$ jump in measles cases worries health officials. Inquirer.Net. [Online]. March 01, 2010 [cited 2012 August]. Available from http://newsinfo.inquirer.net/breakingnews/nation/view/20100301256060/1891-jump-in-measles-cases-worries-health-officials

17. Lapeña CG. Measles cases have more than tripled early this year DOH. GMANetwork.Com. [Online]. March 16, 2010 [cited 2012 August]. Available from http://www.gmanetwork.com/news/story/186246/news/ nation/measles-cases-have-more-than-tripled-early-this-year-doh 